\title{
EROSION OF POPPET VALVE CHAMBER BY HIGH WATER CONTENT FLUID
}

\author{
Teruyuki Maeda*, Soichi Sato** \\ ${ }^{*}$ Faculty of Engineering, Seikei University \\ Tokyo, Japan \\ ${ }^{* *}$ Mitsutoyo Co., Ltd. \\ Kawasaki, Japan
}

ABSTRACT

This paper treats the experimental research of the erosion of the poppet valve chamber by changing the configuration of the poppet valves and seats and the pressure of the poppet valve chamber.

As the result of the experiment, the following became clear: 1) The erosion in the valve chamber is the result of the cavitation and jet. 2) There occur extreamly erosion in the case of the diverging flow than parallel and converging flow. 3) Erosion has its maximum value against the back pressure, and at this time the noise level becomes maximum. 4) Ersion of the valve chamber become small according to an increase in the diameter of the valve chamber. 5) Effective and simple preventive measure of the erosion is presented.

\section{KEYWORDS}

Cavitation, Erosion, HWCF, Preventive measure, Valve chamber.

\section{NOMENCLATURE}

$D$ : diameter of valve chamber [mm] Ea: entrained region of valve chamber

\author{
$\mathrm{p}_{\mathrm{a}}$ : pressure in the entrained region [MPa] \\ $\mathrm{p}_{\mathrm{a}}$ : downstream pressure [MPa] \\ $\mathrm{p}_{\mathrm{u}}$ : inlet pressure of a poppet valve [MPa] \\ $x$ : distance from a valve seat [m] \\ Wa: wall of entrained region \\ Wj: wall where jet impinge on
}

\section{INTRODUCTION}

Taking oil shock opportunity, High Water Contents Fluid (HWCF) which contains water more than 95 percent of the fluid has been investigated vigorously in the world as the working fluid that take the place of mineral oil.

HWCF has an advantage of noninflammable, cheap and clean, but on the contrary they have a great disadvantage of poor lubrication, grouwth of rust and bacterium and occuring of cavitations and so on.

Poppet valves are widely used as the safety valve or main valve of logic valves. Saturated steam pressure of water is very higher than mineral oil and that the cavitation is easy to occur. So, if we use the poppet valve in HWCF as the working fluid, erosion of poppet valve, seat and valve chamber become serious.

In this paper we describe an experimental study of the erosion of the poppet valve chamber by changing the angle of the poppet valve and valve seat and the diameter of the valve chamber and back 
pressure.

2. EXPERIMENTAL APPARATUS AND PROCEDURE

\subsection{Working Fluid And Schematic}

Illustration of Test Setup

IIWCF used in the experiment is the commercial working fluid of Moresco-Aquadol made by Matsumura Oil Research Corporation.

Original solution was diluted with ion exchange water to twenty times. The fluid was exchanged to new one when $\mathrm{pH}$ of the fluid become lower than 8.2. Properties of the working fluid used in the experiments are given in Table 2.1.

Fig.2.1 shows a schematic illustration of the test setup. Fluid is pressurized by an axial displacement piston pump, and fluid flow is regulated by adjusting the valve lift of the test poppet valve by watching a flow meter. Particle by the erosion was

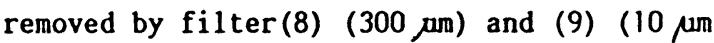
) in Fig.2.1. Noise level was measured by noise meter by accessing to the test valve. Testing conditions are given in Table 2.2.

\subsection{Testing Valves}

Fig. 2.2 shows the testing valve. So as not to be suffered the erosion, the material of the poppet valves and their seats are made SUS- 304, but the valve chamber are made of alminum alloy (A2024BET4) so as to be suffered the erosion easily. A part of the poppet valves and their chamber are shown in Fig.2.3. Here the valve chamber is covered with SUS- 304 not to be scratched at the time of assembling and disassembling.

Table 2.3 shows the combinations of the test to see the effect of the diameter of the valve chamber, the angle of the poppet valves and their seats. To see the effect of the back pressure Pd, it is controlled by a valve (10) in Fig.2.1.

\subsection{Estimation of Erosion}

Washing of the test pieces was done as follows:

(a) Before Test

1) Wash by brush with detergent.

2) Wash in the detergent liquid with ultra-sonic wave for a quarter.

3) Wash by city water with ultra-sonic wave for a quarter.
4) Take off water by Isopropanol.

5) Take off grease by Kerosene Ether.

6 ) Measure the weight of the test pieces by electronic scale (c.clm min.) after decicating about 12

hours in a decicator.

(b) After Test

7) Measure the weight of the test pieces after doing the same procedure except 2) and 3) in the above-mentioned.

8) After dividing the test pieces into halves, observe the state of erosion and take photograph.

Table 2.1 Diluted fluid (20 times)

\begin{tabular}{c|l}
\hline water $(\%)$ & 98.4 \\
specific gravity & 1.0 \\
kinematic viscosity & $0.68 \times 10^{-6}$ \\
$\left(\mathrm{~m}^{2} / \mathrm{s}\right)$ & $\mathrm{pH}$ \\
appearance & emalsion
\end{tabular}

Table 2.2 Tested conaitions

\begin{tabular}{l|l}
\hline differential pressure & \\
$\Delta \mathrm{p}=\mathrm{p}_{\mathbf{\omega}}-\mathrm{p}_{\mathrm{d}}(\mathrm{MPa})$ & 3.73 \\
flow rate $(\mathrm{I} / \mathrm{min})$. & 38 \\
testing hours (hrs) & 5 \\
testing temperature $\left(\mathrm{C}^{\circ}\right)$ & $40+0.5$
\end{tabular}

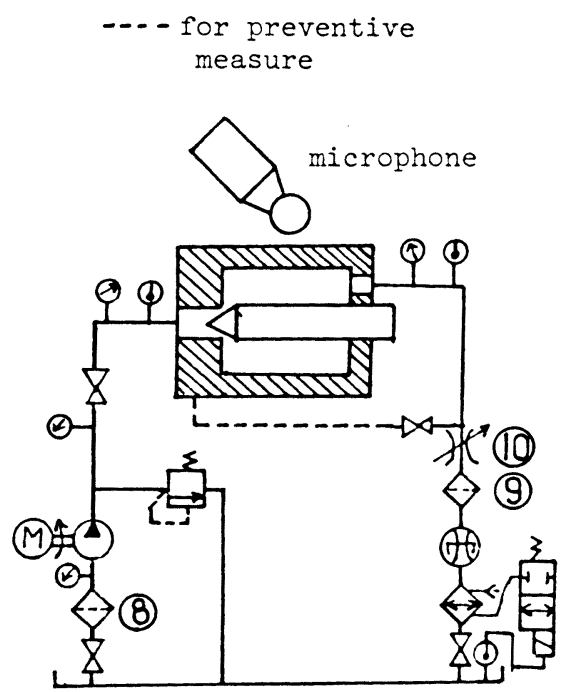

Fig.2.l Schematic illustration of test setur 


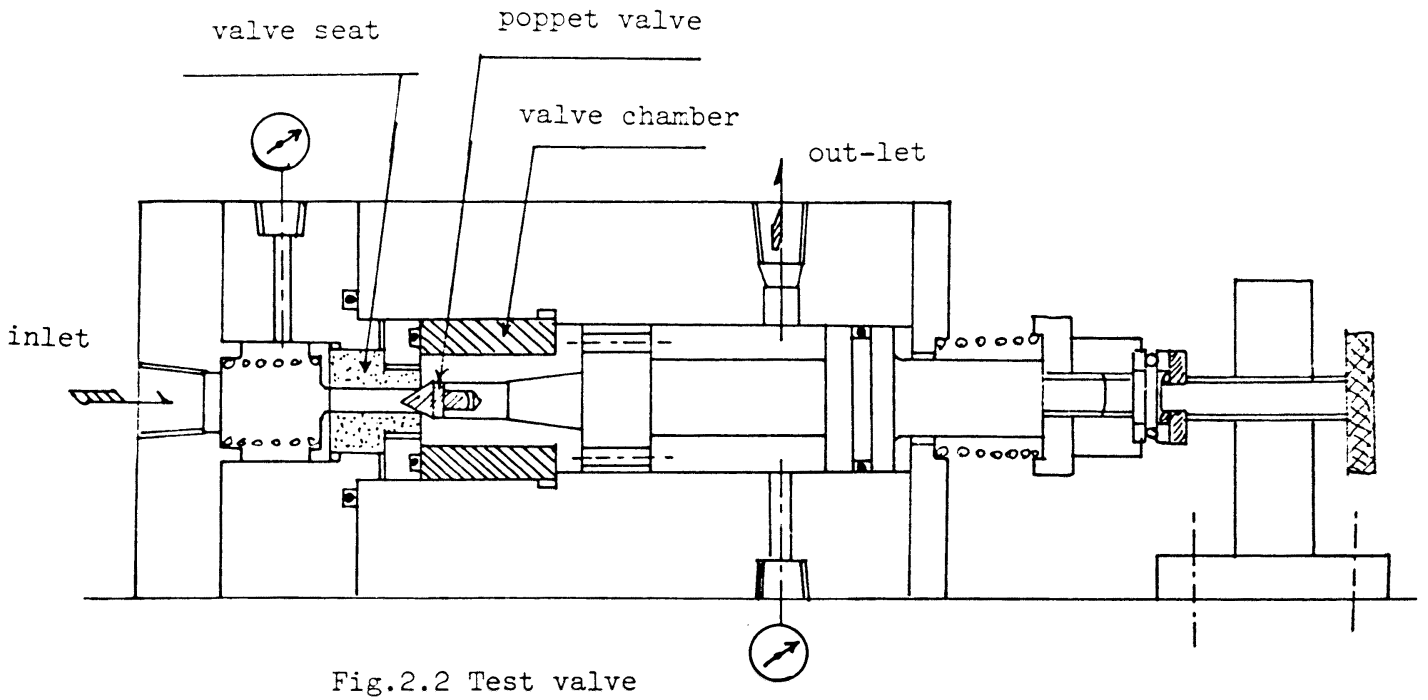

Table 2.3 Combination of test pieces

\begin{tabular}{c|c|c|c}
\hline & $\alpha^{\circ}$ & $\beta^{\circ}$ & $D \mathrm{~mm}$ \\
\hline $\begin{array}{c}\text { to check the effect } \\
\text { of D.V.C. }\end{array}$ & 45 & 60 & $\begin{array}{c}20 \\
24\end{array}$ \\
\hline & 30 & & \\
to check the effect & 60 & 60 & 20 \\
of A.P.V. & 90 & & \\
\hline to check the effect \\
of A.V.S.
\end{tabular}

D.V.C.: diameter of valve chamber A.P.V.:angle of poppet valve A.V.S.:angle of valve seat

\section{Experimental Results And Considerations}

In this paper, we call the relationship between valve seat angle $\beta$ and poppet valve angle $\alpha$ as follows:

: diverging flow when $\beta>\alpha$

: parallel flow when $\beta=\alpha$

: converging flow when $\beta<\alpha$

3.1 Effect of The Diameter of Valve
Chamber

Fig.3.1-a,b,c show the amount of erosion, noise level and the location of erosion of the valve chamber changing the diameter of the valve chamber $D \mathrm{~mm}$ tol4, 20,24 and back pressure in the case of the diverging flow $\left(\alpha=45^{\circ}, \beta=60^{\circ}\right)$.

From the figures we can see as follows:

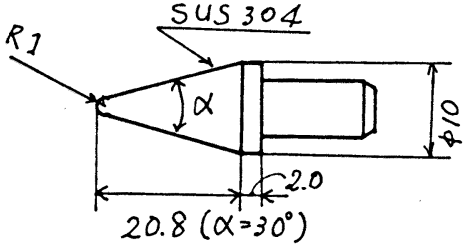

(a) poppet valve $\left(\alpha^{\circ}=30,45,60,90\right)$

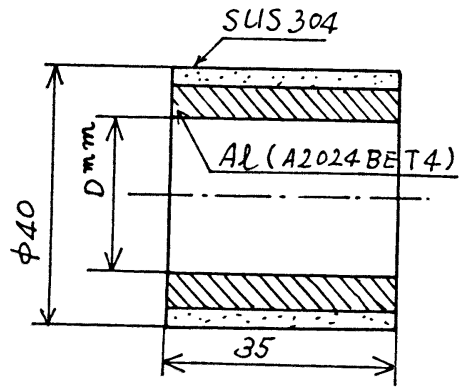

(b) valve chamber $(D=14,20,24)$

Fig. 2.3 Poppet valve and valve chamber

1) An increase in the diameter result in a decrease in the erosion.

2) According to an increase in the back pressure result in an increase in the erosion and that shows its maximum at some back pressure. Moreover, if we increase the back pressure in succession, the erosion come to decrease and finally there are no erosion to recognize at the valve chamber.

3) Noise level has the same chacteristics as the erosion and the back preasure in which the maximum erosion occur coincide with the one of the maximum noise. 


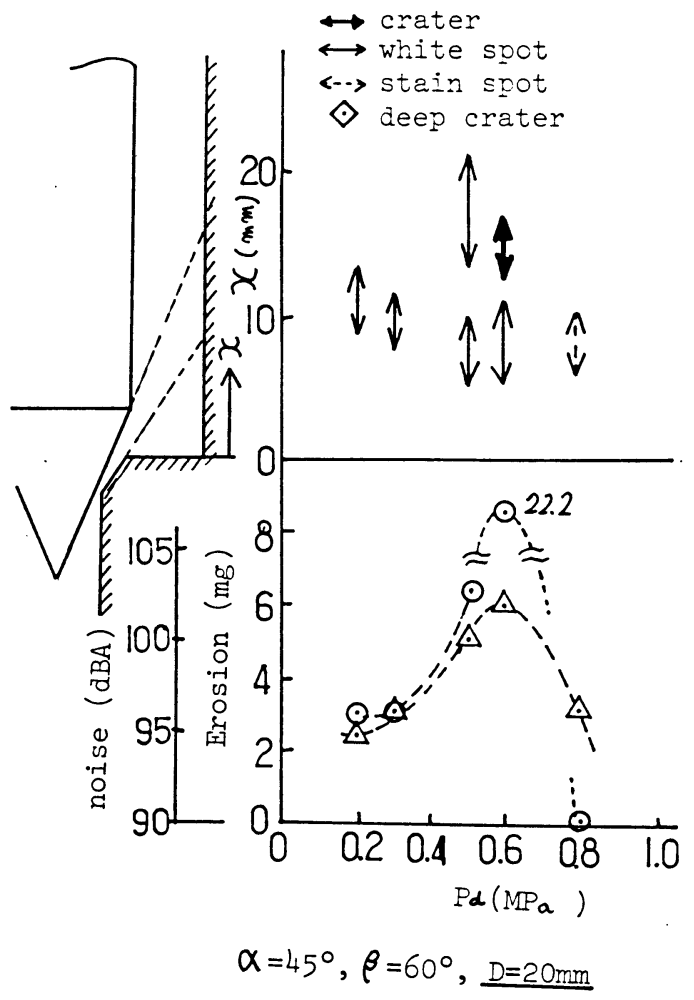

Fig.3.1-a Effect of the diameter of the valve chamber

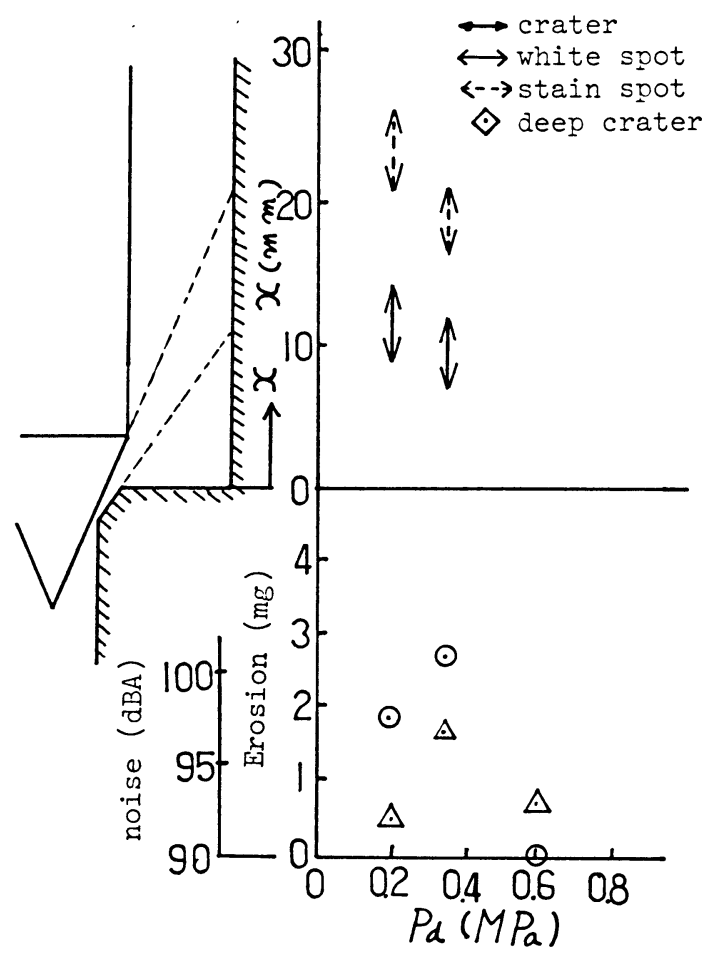

$\alpha=45^{\circ}, \beta=60^{\circ}, \underline{D=24 \mathrm{~mm}}$

Fig.3.1-c Effect of the diameter of the valve chamber

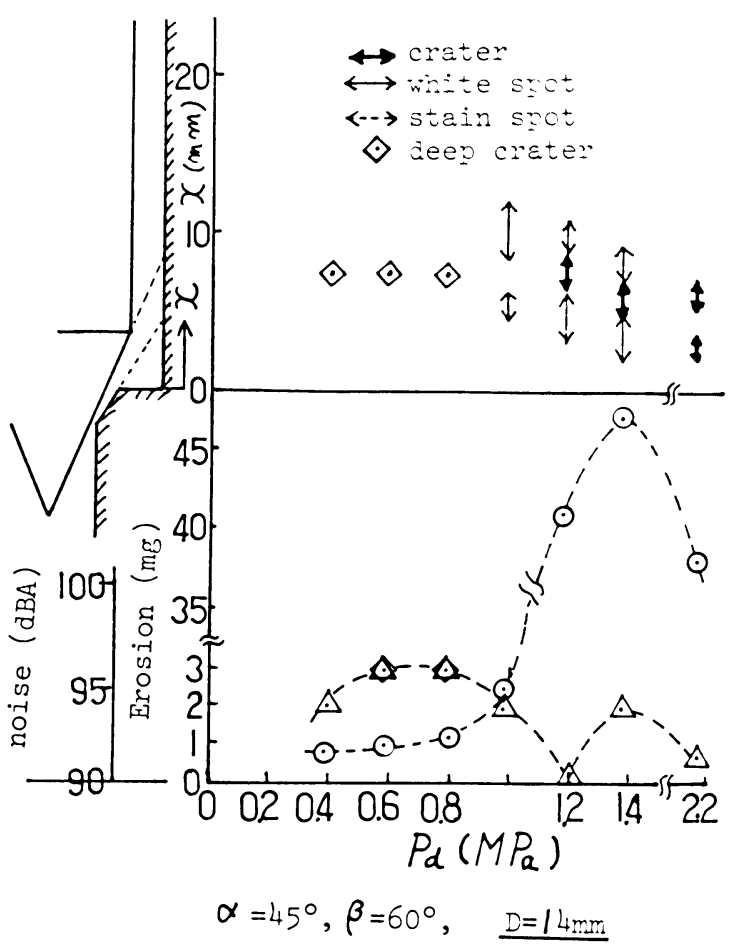

Fig.3.1-b Effect of the diameter of the valve chamber

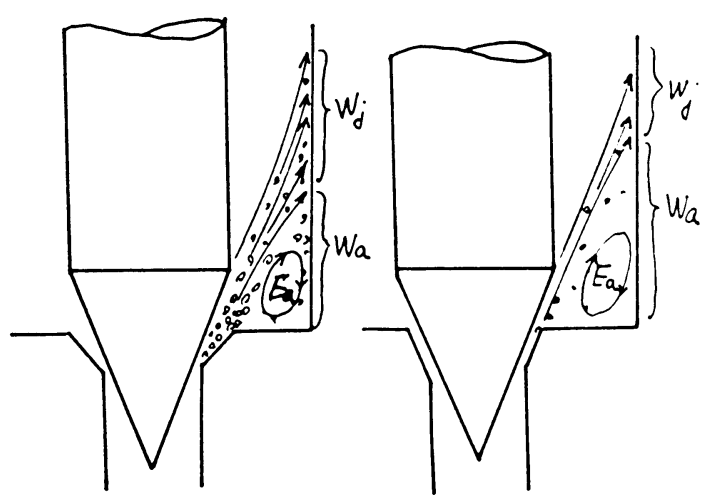

(a) diverging flow (b) parallel flow

Fig.3.2 Schematic illustration of the flow pattern in the valve chamber

4) According to an increase in the back pressure result in an approach of the occuring location of the erosion to the valve seat side. The reason of this phenomenon is discussed in 3.3.

5) As shown in Fig.3.2-a, locations of the erosion are divided into the region indicated by $W j$ where the jet impinges on and the jet makes crater and the region of the valve seat side indicated by Wa and the downstream side where the surface of the valve chamber wall changes its colour white by the erosion. 
3.2 Diverging Flow, Parallel Flow And Converging Flow

Fig.3.3-a shows the maximum erosion of the valve chamber against $\alpha$ where $D=20 \mathrm{~mm}$ and $\beta=60^{\circ}$.

Fig.3.3-b shows the maximum erosion of the valve chamber against $\beta$ where $D=20 \mathrm{~mm}$ and $\alpha=45^{\circ}$.

It is clear from the figures that the erosion are extremely large at the diverging flow and that an increase in $(\beta-\alpha)$ results in an increase in the erosion.

In the cases of parallel flow and converging flow, the erosion are very small and there are no difference between them.

Fig.3.4 shows the locations of the erosion of the valve chamber when the maximum erosion occured at the same conditions of Fig.3.3.

It can be seen from the figures as follows:

1) In the case of the diverging flow, there occurs the erosion of a sort of crater around the wall where the jet along the

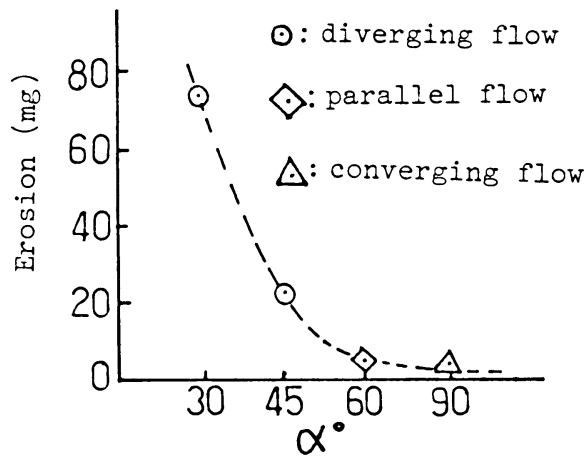

(a) Effect of angle of poppet valve $\left(\beta=60^{\circ}, D=20 \mathrm{~mm}\right)$

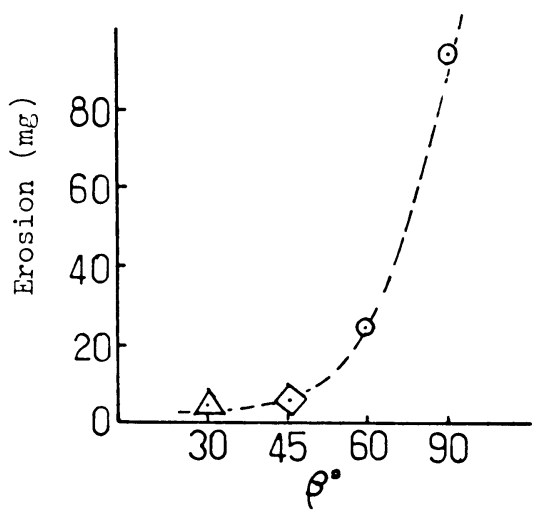

(b) Effect of angle of valve seat $\left(\alpha=45^{\circ}, D=20 \mathrm{~mm}\right)$

Fig.3.3 Comparison of maximum erosion poppet valve impinges on. This is due to cooperation with the jet and the cavitation erosion

On the contrary in the cases of the parallel and converging flow, as a less quantity of cavities are produced than the diverging flow, a small number of cavities are transfered to the wall and sweep out to the downstream, there occurs less erosion.

2) We can see the spot changed white at the wall Wa. This is due to the cavitation erosion by the cavities which are entrained in this region.

3) In the case of the diverging flow, we can see the erosion at the downstream side of Wj. On the other hand, in the case of the

$\leftrightarrow$ crater

$\longleftrightarrow$ white spot $\leftrightarrow$ stain spot $A$ flow along poppet valve $\rightarrow$ flow along valve seat

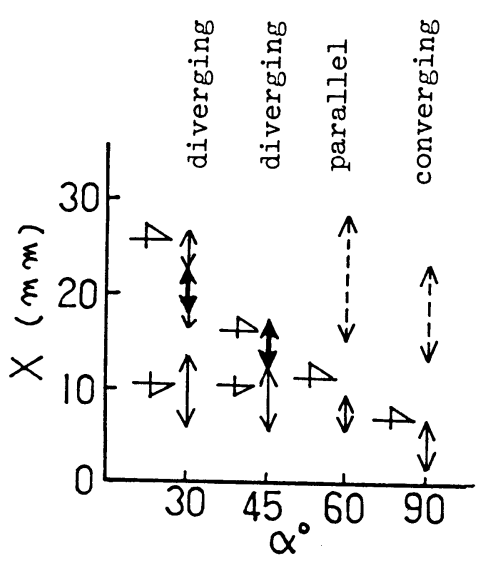

(a) Effect of angle of poppet valve $\left(\beta=60^{\circ}, D=20 \mathrm{~mm}\right)$

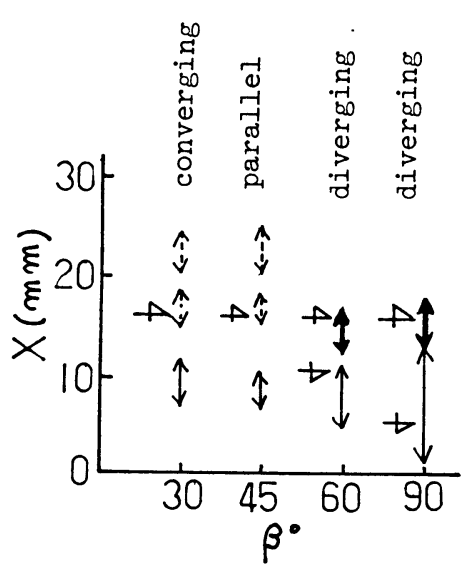

(b) Effect of angle of valve seat $\left(\alpha=45^{\circ}, D=20 \mathrm{~mm}\right)$

Fig. 3.4 Location of erosion when maximum erosion occured 
parallel and the converging flow, we can not see the erosion at the downstream side of wj.

\subsection{Effect of Downstream Pressure}

We can see the following facts from Fig.3.1-a,b,c about the effect of the downstream pressure Pd.

1) An increase of the downstream pressure result in a shifting of the location of the erosion damage to the valve seat side. This is, as shown in Fig.3.2, due to deflected jet toward the valve seat side by the pressure difference between high pressure of

the downstream side and low pressure of the valve seat side.

2) As mentioned in 3.1(2), according to an increase in the back pressure result in an increase in the erosion and it shows its maximum erosion at some back pressure, and

finally it disappear by increasing the back pressure in succession. This is due to (a) shorten distance to the wall by the deflection of the jet toward the valve seat side and (b) just at this back pressure, cavities are collapsed at the wall.

If we increase the back pressure in succession, the cavities are collapsed before they transfer to the wall, so we can not see the cavitation erosion at this time.

Photograph 3.1 show the cut test pieces.

4. An Example of Preventive Measure of Erosion

On the ground of the experiment, it may be quite all right to consider the occuring of the erosion in the valve chamber is by the collapse of the cavity at the wall which stagnated in the entrained region $\mathrm{Ea}$ and the impinging to the wall $\mathrm{Wa}$ and $\mathrm{Wj}$. Therefore, we can propose an example of preventive measure of erosion as follows. These are 1) to get rid of the entrained region Ea. 2) to make a flow at the entrained region to sweep out the cavities to the downstream.

In this paper, we tested method 2). Fig.4.1 shows a preventive measure of erosion tested in this experiment. According to the feedback of the downstream pressure to the valve seat side of the valve chamber, there occur the flow in this loop because of $\mathrm{Pd}>\mathrm{Pa}$ and the deflection of the jet toward the downstream side. So we can expect the sweep out of the cavities from the entrained region.

Fig.4.2 shows the experimental result of with preventive measure and without preventive measure. We can see from the figure as follow:

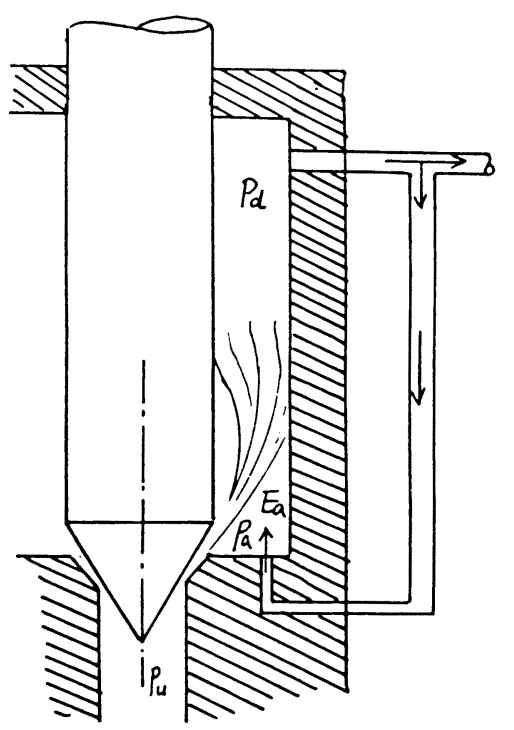

Fig. 4. 1 Preventive measure of erosion

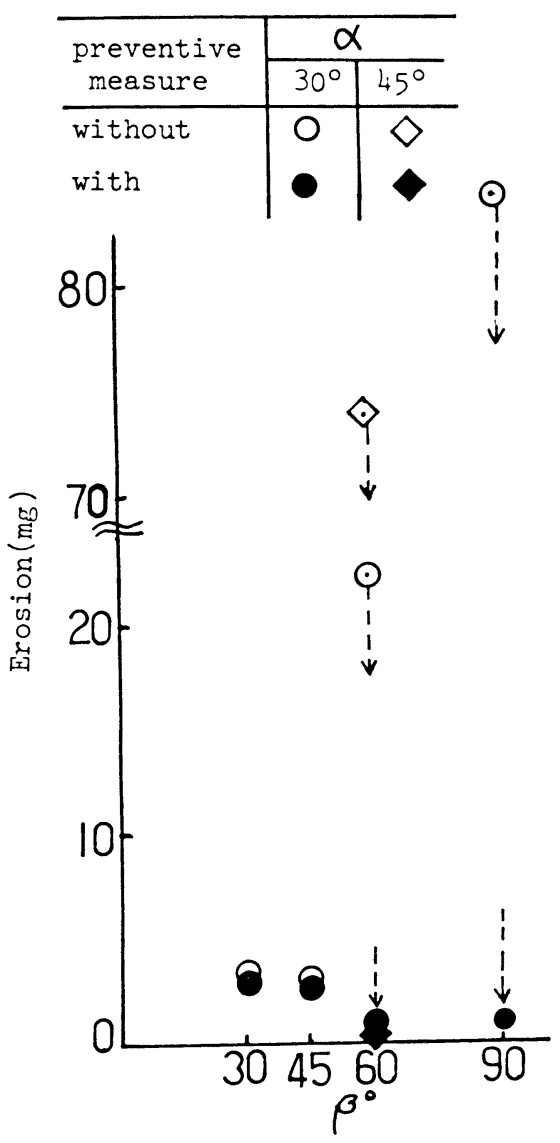

Fig.4.2 Comparison of erosion of with preventive and without preventive measure 
1) In the case of the diverging flow, there are no erosion of a sort of crater except a stain spot by adopting the preventive measure.

2) In the case of the parallel and the converging flow, there are almost no effect of the preventive measure. This can be considered that the discrepancy between them don't appear because of the erosion in the case of without preventive measure is small enough.

Photograph 4.1 show the cut test pieces of the with preventive and without preventive measure.

\section{Conclusions}

In this paper the authors made an experimental analysis on the erosion of poppet valve chamber with HWCF and its preventive measure. From the facts described above, we may conclude as follows:

1) The location of the erosion at the valve chamber wall are divided into three regions, that is entrained region wall Wa, wall where the jet impinges and the downstream region.

2) Erosion takes its maximum against the downstream pressure.

3) There occur much erosion in the case of the diverging flow than the parallel and converging flow.

4) Erosion decreases rapidly as the diameter of the valve chamber increases.

5) Effective and simple preventive measure of the erosion is presented.

\section{REFERENCES}

(I) Hibi,A., Ichikawa, T., Miyakawa, S., :Flow Characteristics of Cyrindrical Chokes, J. Japan Hydraulics Phneumatics Soc. (in Japanease), Vol.2, No.2, | 971 -4, pp8- 6.

(2) Yamaguchi, J.: Cavitation of Working Fluid, J.Japan Hydraulics Phneumatics Soc. (in Japanease), Vol.5, No.2, /974, pp79-85.

(3) Iwaizumi,Y., et al. : Research on Fluid Power Systems for use with HighWater Based Fluid, Institute of Technology of Kikai Shinko Association, KSK-G58. , I 984.

(4) Ichikawa,T., : Cavitation of Fluid Power Systems, J.Japan Hydraulics Phneumatics Soc. (in Japanease), Vol. 6, No.5, 1985, pp43-49.
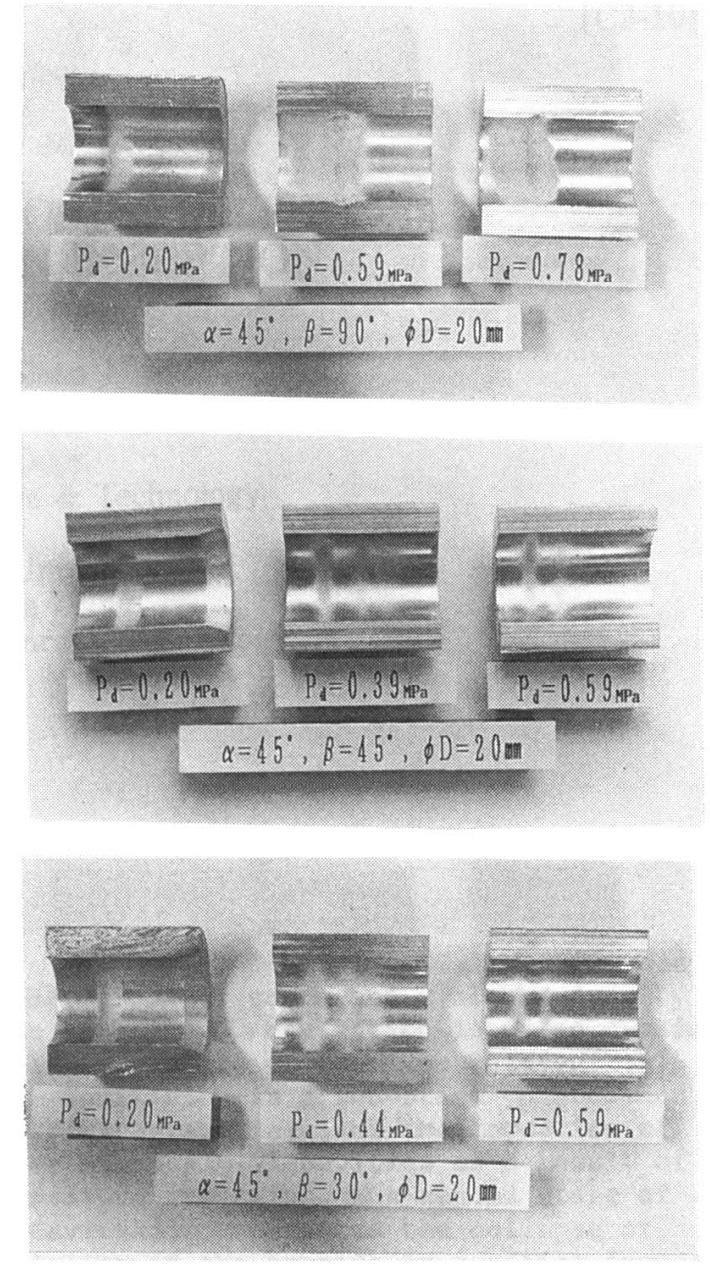

Photo 3.1 Effect of downstream pressure

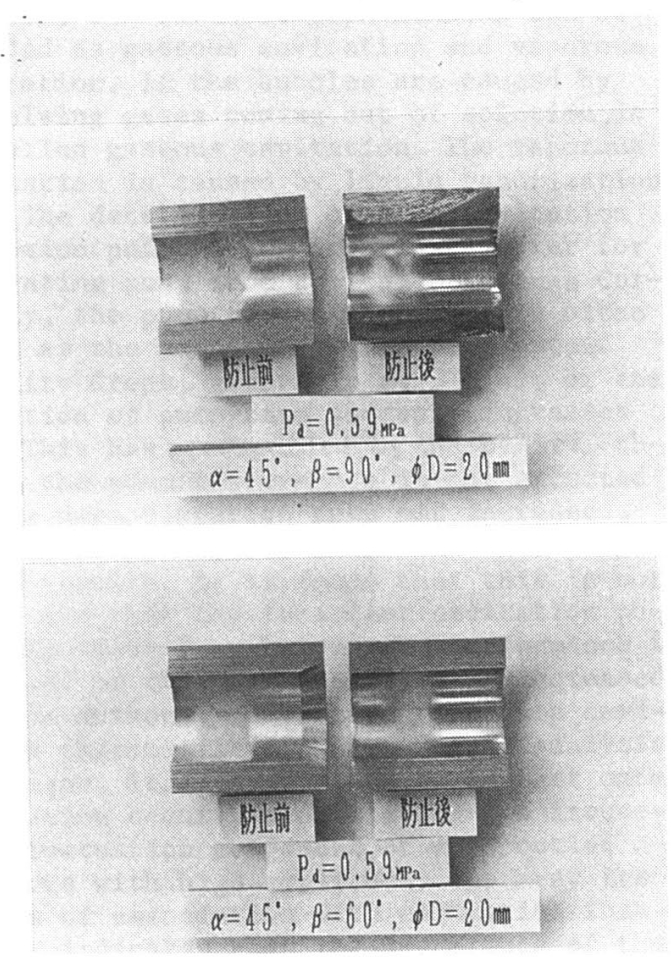

Photo 4.1 Test pieces after test with preventive and without preventive measure 